\title{
A General Circulation Model Study of the Impact of a Stratospheric Sudden Warming Event on Tropical Convection
}

\author{
Kunihiko Kodera $^{1,2}$, Hitoshi Mukougawa ${ }^{3}$, and Yuhji Kuroda ${ }^{2}$ \\ ${ }^{1}$ Sorlar-Terrestrial Environment Laboratory, Nagoya University, Nagoya, Japan \\ ${ }^{2}$ Meteorological Research Institute, Tsukuba, Japan \\ ${ }^{3}$ Disaster Prevention Research Institute, Kyoto University, Kyoto, Japan
}

\begin{abstract}
The impact of a Northern Hemisphere stratospheric sudden warming (SSW) event on tropical convection is investigated as an initial value problem using a general circulation model. The amplification of planetary waves that produced the simulated SSW was forced by introducing an anomalous field in the model initial conditions. The impact of this SSW event on the tropics was examined by comparing runs with and without the SSW. An increased stratospheric mean meridional circulation cools the tropical lower stratosphere when planetary waves propagate upward into the stratosphere, leading to enhanced convective activity in the equatorial Southern Hemisphere. This result is consistent with previous observational studies, and indicates that convective activity in the equatorial region is influenced by SSW events.
\end{abstract}

\section{Introduction}

It has long been known that stratospheric sudden warming (SSW) events induce cooling in the tropical stratosphere due to changes in the hemispheric meridional stratospheric circulation (Julian and Labitzke 1965; Fritz and Soules 1970). These circulation changes are induced by amplified planetary wave forcings (Dunkerton et al. 1981; Garcia 1987; Randel 1993). Recent studies have suggested that the impact of SSW events penetrates still further downward in the tropics, by modulating convective activity in the tropical troposphere (Kodera 2006; Eguchi and Kodera 2010; Kuroda 2008). However, tropical convection can influence the stratospheric circulation through modification of the extratropical planetary waves (Taguchi and Hartmann 2006), or tropical planetary waves (Norton 2006). The stratosphere and troposphere have their own variability, but they are also closely coupled. It is, therefore, very difficult to identify whether tropical convection triggers the SSW event or vice versa from observational studies alone.

In this study we have conducted numerical experiments using a general circulation model to more clearly determine causality in the relationship between SSW events and changes in tropical convection. The present experiment introduces a tropospheric blocking-type anomaly field in the model initial conditions to force the SSW. We compare two ensembles of simulations, one with an SSW event and the other without, to identify how the influence of the extra-tropical troposphere is transmitted to the tropical troposphere.

\section{Model and experimental method}

Anomalous circulation patterns responsible for the occurrence of the SSW have been identified by means of the regression of polar stratospheric temperatures in forecast experiment of the SSW of December 2001 (Fig. 3 in Mukougawa et al. 2005). This

Corresponding author: Kunihiko Kodera, Sorlar-Terrestrial Environment Laboratory, Nagoya University, Furo-cho, Chikusa-ku, Nagoya 464-8601, Japan. E-mail: kodera@stelab.nagoya-u.ac.jp. C2011, the Meteorological Society of Japan. circulation pattern is incorporated as a dynamical seed in the model initial conditions north of $20^{\circ} \mathrm{N}$. A SSW is generated by the model approximately two weeks into the model run. The SSW can be suppressed by reversing the polarity of the seed anomaly. We further superimpose small perturbations to the initial conditions to construct 13-member ensembles. These initial perturbations were obtained from the Japan Meteorological Agency (JMA) multimonth ensemble forecast system for 13 December 2001. Perturbations are added to all pressure levels north of $20^{\circ} \mathrm{N}$. The amplitude of the perturbations is set to $14.5 \%$ of the climatological rootmean-square variance in $500 \mathrm{hPa}$ geopotential heights. The results are reported as ensemble means.

The model that we use is a general circulation model jointly developed by the JMA and Meteorological Research Institute (MRI) (Mizuta et al. 2006), and is identical to that used by Mukougawa et al. (2007). Experiments are conducted on a linear Gaussian grid with triangular truncation 96 (TL96) and 40 vertical levels with model top at $0.4 \mathrm{hPa}$. (Corresponding horizontal resolution is about $200 \mathrm{~km}$. Vertical resolution varies according to the height. The model includes six levels near the tropical tropopause between 200- and $90 \mathrm{hPa}$.) Fixed sea surface temperature (SST) anomalies corresponding to those observed at the initial time (13 December 2001) are superimposed onto the climatological SST as lower boundary conditions.

\section{Results}

Figure 1a shows ensemble averages of mean geopotential height on day 0 for the no-SSW (left panel) and SSW (right panel) ensembles. Geopotential heights at $10 \mathrm{hPa}$ are displayed in the top panels, and geopotential heights at $500 \mathrm{hPa}$ in the bottom panels. Differences between the SSW and no-SSW ensembles are indicated by the shading in the right panels. The daily mean differences on day 0 effectively represent differences in the "seed" anomaly introduced in the initial conditions. Euro-Atlantic blocking type anomalies are prominent at $500 \mathrm{hPa}$ in the SSW ensemble, whereas the $500 \mathrm{hPa}$ flow is zonal in the no-SSW ensemble. The stratospheric polar vortex at $10 \mathrm{hPa}$ is of similar strength in both ensembles on day 0 , although the Aleutian high is slightly more developed in the SSW ensemble. After two weeks, the stratospheric polar vortex is substantially weakened and displaced over Europe in the SSW ensemble due to strong polar warming. The stratospheric polar vortex remains strong and cold on day 14 in the no-SSW ensemble.

Figure 2a shows the time evolution of daily mean $10 \mathrm{hPa}$ air temperatures at $80^{\circ} \mathrm{N}$ for the first 14 days of each member simulation. All 13 member simulations incorporating the positive seed anomaly produce a SSW (red lines), while none of the 13 member simulations incorporating the negative seed anomaly produces a SSW (blue lines). The two ensembles are well separated.

Differences between the SSW and no-SSW ensemble means $(\Delta)$ are shown in Fig. 2b-e. The statistical significance of these differences is evaluated using the Student's $t$ test. All of the differences discussed here is significant at the 95\% level. To preserve the clarity of the figures, statistical significance is indicated only in Fig. $2 \mathrm{c}-$ e. Shading indicates regions for which the differences are not significant at the $95 \%$ level. Figure $2 \mathrm{~b}$ shows differences in the Eliassen-Palm (E-P) flux at $70 \mathrm{hPa}$. The vertical and horizon- 
(a) day 0
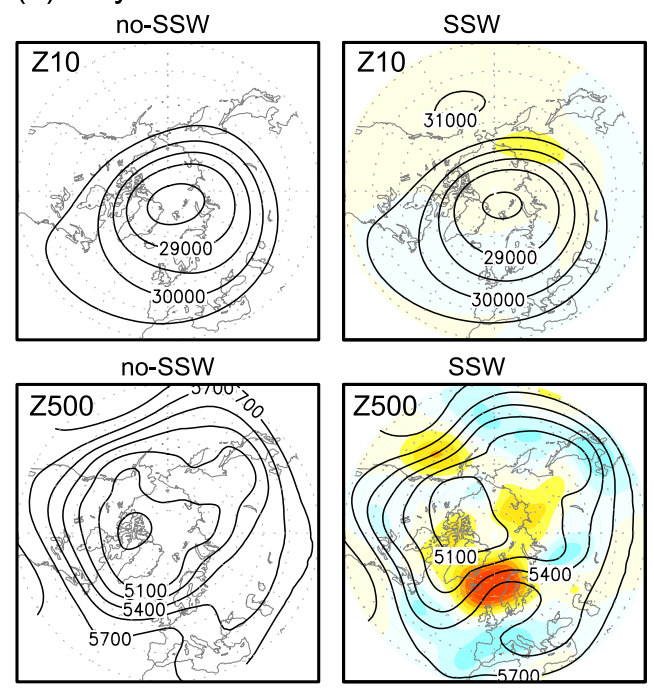

(b) day 14
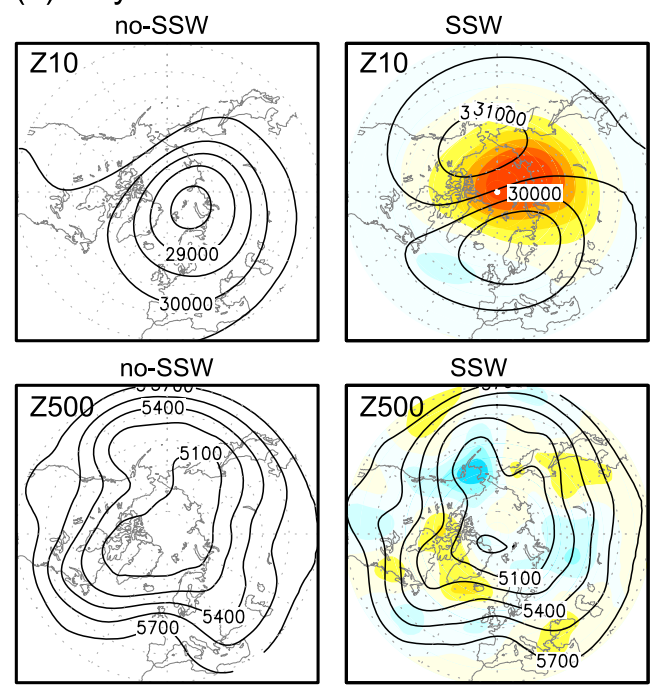

$\Delta \mathrm{Z}: \mathrm{SSW}-\mathrm{no} \mathrm{SSW}$

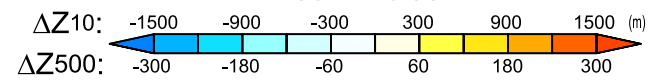

Fig. 1. (a) Ensemble averages of the daily mean geopotential height [m] at $10 \mathrm{hPa}$ (top) and $500 \mathrm{hPa}$ (bottom) on day 0 for the no-SSW (left) and SSW (right) ensembles. The difference between the two ensemble means $(\Delta \mathrm{Z})$ is indicated by colored shading in the right panels. (b) As in (a) but for day 14.

tal components of the E-P flux are displayed using color shading and contours, respectively. Amplified waves propagate horizontally southward into the subtropics at around $30^{\circ} \mathrm{N}$ at first, before upward propagation along the polar night jet (near $\left.60^{\circ} \mathrm{N}\right)$ starts at around day 3. Equatorward propagation of waves in the lower stratosphere diminishes and upward propagation of planetary wave into the upper stratosphere increases after day 8. After the polar warming matures, vertical wave propagation weakens and poleward propagation strengthens from day 11. Usually, stratospheric polar warming lags the equatorial cooling because the planetary waves propagate equatorward before becoming focused over high latitudes as described in a previous paper (Kodera et al. 2011).

These changes in wave propagation are accompanied by changes in the tropical upwelling branch of the wave-driven meridional circulation. Figure $2 \mathrm{c}$ shows a latitude-time section of the zonal-mean pressure-coordinate vertical velocity $(\omega)$ at $50 \mathrm{hPa}$.
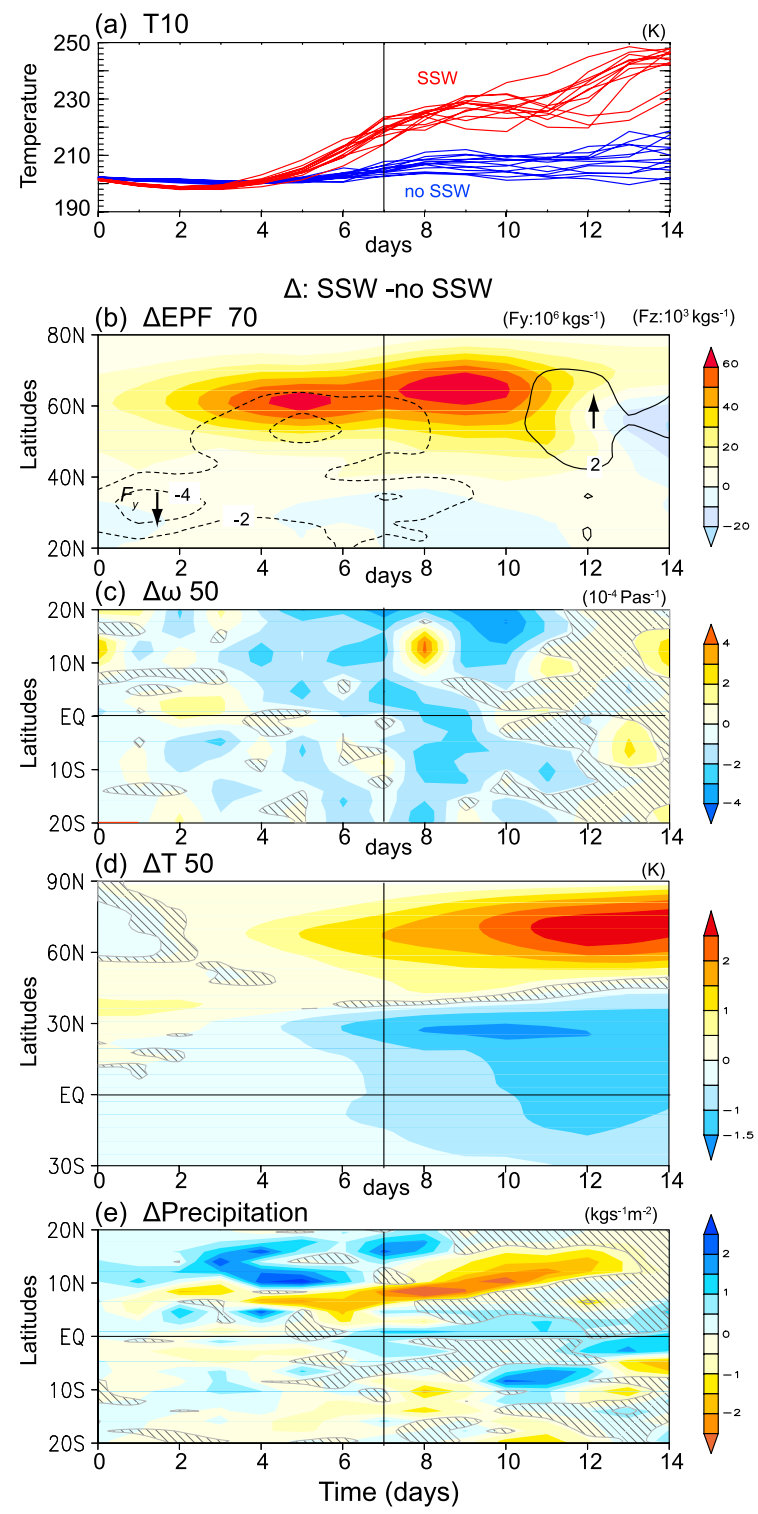

Fig. 2. (a) Time series of $10 \mathrm{hPa}$ air temperature at $80^{\circ} \mathrm{N}$ for each member of the SSW ensemble (red lines) and each member of the no-SSW ensemble (blue lines). (b) Differences in the latitude-time section of Eliasson-Palm (E-P) fluxes at $70 \mathrm{hPa}$ between the SSW and no-SSW ensembles, weighted by cosine of latitude. The vertical component of the $\mathrm{E}-\mathrm{P}$ flux is indicated by color shadings $\left[10^{4} \mathrm{~kg} \mathrm{~s}^{-2}\right]$, and the meridional component by contours $\left[10^{7} \mathrm{~kg} \mathrm{~s}^{-2}\right]$. Arrows indicate the direction of horizontal wave propagation. Zero contour lines are omitted. (c) As in (b), but for pressure-coordinate vertical velocity $(\omega)$ at $50 \mathrm{hPa}\left[\mathrm{Pa} \mathrm{s}{ }^{-1}\right]$. (d) As in (b), but for zonal-mean temperature at $50 \mathrm{hPa}[\mathrm{K}]$, weighted by cosine of latitude. (e) As in (b), but for zonal-mean precipitation rate $\left[\mathrm{kg} \mathrm{m}^{-2} \mathrm{~s}^{-1}\right]$. Shading in panels (c), (d), and (e) indicates that the difference is not statistically significant at the $95 \%$ level.

Note that Figs. 2c and 2e illustrate only the tropical region from $20^{\circ} \mathrm{S}$ to $20^{\circ} \mathrm{N}$. Tropical upwelling intensifies starting from day 4 in the Northern Hemisphere $(\mathrm{NH})$ and from day 8 in the Southern Hemisphere $(\mathrm{SH})$. This enhanced upwelling produces stronger adiabatic cooling in the tropical lower stratosphere; accordingly, the zone of low temperatures at $50 \mathrm{hPa}$ extends from the subtropical $\mathrm{NH}$ to the SH (Fig. 2d). The enhanced tropical upwelling disappears when the wave forcing vanishes, at around day 11 (Fig. 2c). The evolution of the zonal-mean difference in tropical precipitation between the two ensembles is depicted in Fig. 2e. Precipitation in the SSW ensemble increases in the $\mathrm{NH}$ tropics $10^{\circ} \mathrm{N}-20^{\circ} \mathrm{N}$ at first, then decreases after day 8 . The zone of sup- 
$\Delta$ Stream line and $\Delta$ Temperature diff.
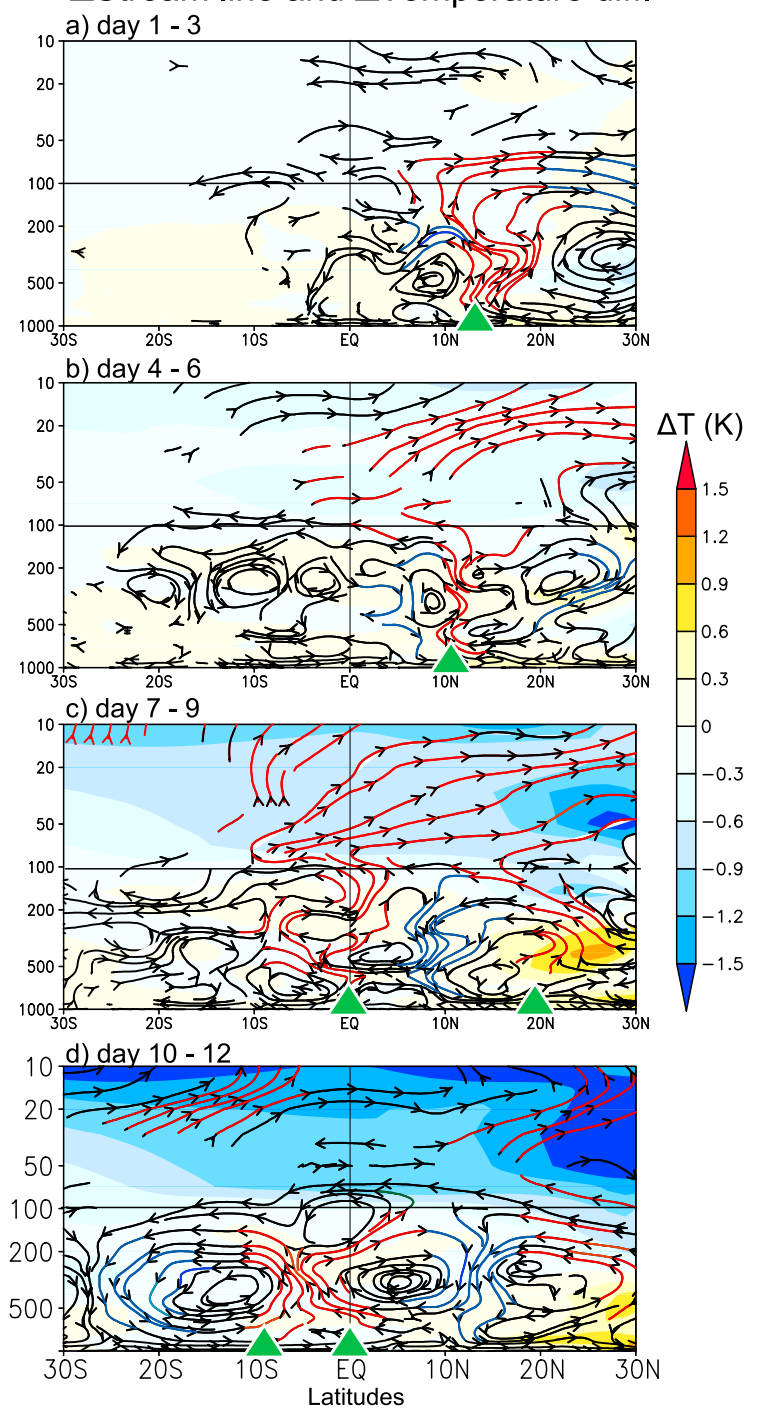

Fig. 3. Three-day-mean differences in zonal-mean temperature (colored shading) and the direction of the mean meridional circulation (streamlines) between the SSW and no-SSW ensembles: (a) days 1-3, (b) days 4-6, (c) days $7-9$, and (d) days $10-12$. Green triangles indicate the approximate center of the precipitating zones identified in Fig. 2e.

pressed precipitation in the $\mathrm{NH}$ then gradually shifts northward, while precipitation over the equatorial $\mathrm{SH}$ increases.

Differences in the three-day-average zonal-mean meridional circulation stream lines between the SSW and no-SSW ensembles are displayed in Fig. 3, along with differences in zonal mean temperature. For convenience, the typical location of the upward branch is indicated by red lines and the typical location of the downward branch by blue lines. The green triangles in Fig. 3 indicate the approximate centers of the precipitating zones identified in the troposphere in Fig. 2e. At the beginning (days 1-3), upwelling increases in the tropical $\mathrm{NH}\left(10^{\circ} \mathrm{N}-20^{\circ} \mathrm{N}\right)$. This increase is related to an enhanced poleward flow near the tropopause level, which is consistent with the initial increase in equatorward wave propagation in the subtropics shown in Fig. 2b. During days 4-6, enhanced upwelling develops in the equatorial lower stratosphere and tropical tropopause layer (TTL) in the NH. Stratospheric upwelling intensifies in the $\mathrm{SH}$ and the tropical stratosphere cools during days 7-9, while enhanced tropospheric upwelling emerges around the equator. The enhanced upwelling in the $\mathrm{NH}$ located near $10^{\circ} \mathrm{N}$ during days $4-6$ shifts northward and the downwelling develops at this point near $10^{\circ} \mathrm{N}$.
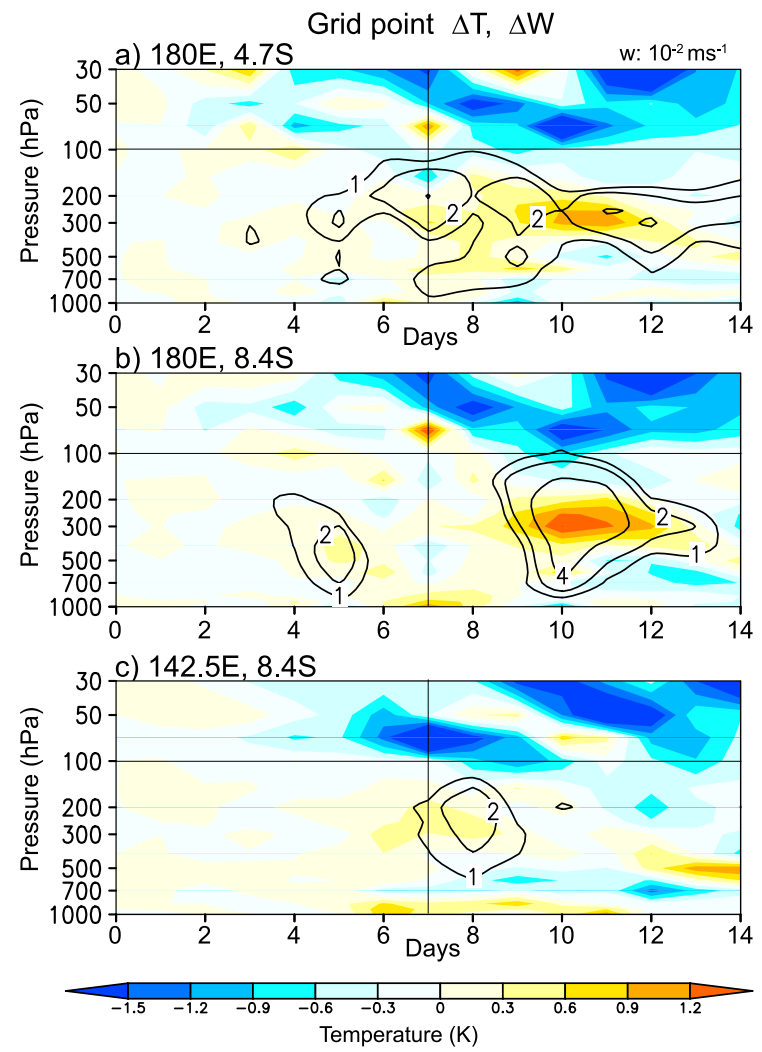

Fig. 4. Height-time sections of differences in daily mean temperature (color shading) and vertical velocity (contours) between the SSW and no-SSW ensembles at model grid points: (a) $180^{\circ} \mathrm{E}, 4.9^{\circ} \mathrm{S}$; (b) $180^{\circ} \mathrm{E}, 8.4^{\circ} \mathrm{S}$; and (c) $142.5^{\circ} \mathrm{E}, 8.4^{\circ} \mathrm{S}$. Vertical velocity contours represent $0.01,0.02$, and $0.04 \mathrm{~m} \mathrm{~s}^{-1}$.

During days 10-12, the stratospheric upwelling over most of the tropics tends to weaken, and the connections between the stratospheric and tropospheric circulations become less clear. In the troposphere, upwelling develops near $5^{\circ} \mathrm{S}$ and downwelling develops near $15^{\circ} \mathrm{N}$, indicating a southward shift of the upward branch of the Hadley circulation. Development of similar structures during SSW events has been noted by observational studies (Eguchi and Kodera 2010; Kodera et al. 2011).

A previous study has suggested that stratospheric changes and deep convective activity interact locally (Eguchi and Kodera 2010); therefore, close examination of local aspects is necessary to better understand the connection. The evolutions of differences in temperature and vertical velocity are displayed for three grid points in the equatorial SH in Fig. 4. For clarity, only upwelling that is directly related to convective activity is shown (contours), while both signs of temperature anomalies are shown as colored shading.

Stratospheric cooling becomes apparent in the SSW ensemble after day 7 (indicated in Fig. 4 by vertical lines), consistent with the zonal mean features shown in Fig. 2; however, the local temperature field also includes wave-like variations that supplement this gradual cooling tendency. As low temperature anomalies descend to the $100 \mathrm{hPa}$ level, upwelling in the TTL increases. These changes in TTL upwelling are accompanied by increases in temperatures in the upper troposphere $200-300 \mathrm{hPa}$ layer.

Activation of the deep convective activity is not limited to the three points. Figure 5 shows differences in temperature and vertical velocity that are similar to those in Fig. 4, but for all grid points along $8.4^{\circ} \mathrm{S}$ for day 5 and day 9 . As the stratospheric cooling develops, upwelling and temperature increase in the upper troposphere and TTL over a wide longitude range. 

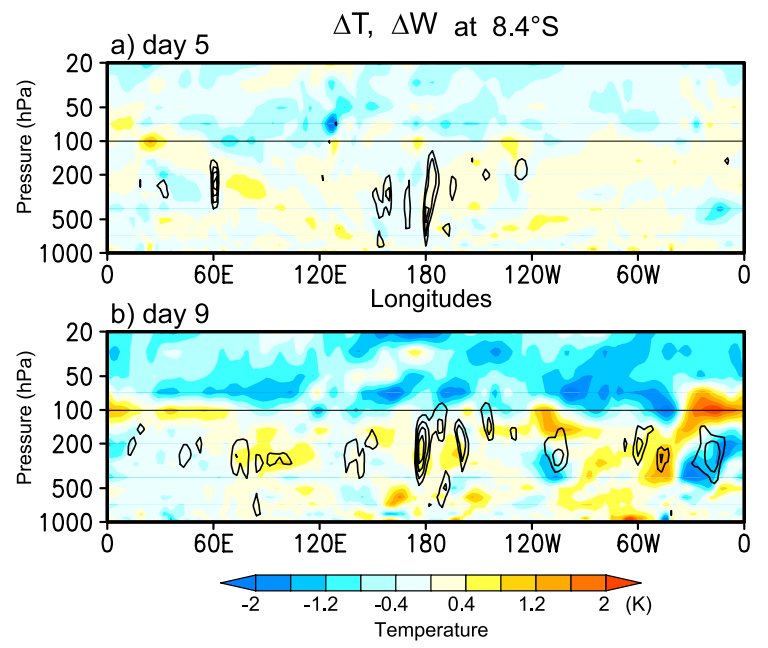

Fig. 5. Height-longitude sections along $8.4^{\circ} \mathrm{S}$ of differences in daily mean temperature (color shading) and vertical velocity (contours) between the SSW and no-SSW ensembles on (a) day 5 and (b) day 9. Only upward velocity is displayed. The contour interval is $0.01 \mathrm{~m} \mathrm{~s}^{-1}$.

\section{Concluding remarks}

We have conducted numerical experiments to clarify the causal relationship between a SSW event and changes in tropical circulation and convection. The southward shift of convective activity in the tropics following a NH SSW reported by a previous observational study (Kodera 2006) occurs in our model experiment. Precipitation in the tropical $\mathrm{NH}$ is enhanced at first, from days $2-6$ of the integration, and then diminished (see Fig. 2e). The initial increase of precipitation in the $\mathrm{NH}$ is excited by changes in the meridional circulation near the tropopause level (Fig. 2), while the enhancement of convective activity in the equatorial SH after day 7 of the integration is induced by changes in the large-scale stratospheric mean meridional circulation.

Tropospheric Rossby waves generated over the Euro-Atlantic region, where the anomalous blocking-type field is introduced in our model initial conditions, may excite convective activity over the tropical Indian Ocean when conditions are favorable for wave propagation into the tropics along the tropospheric subtropical jet (Hsu et al. 1990; Inaba and Kodera 2010). No such effect was identified in the present model simulations. The activation of tropical convection occurs over a wide longitudinal range, and is associated with cooling in the tropical stratosphere (Fig. 5).

It has been argued that the impacts of the stratospheric planetary wave forcing are too small to account for the tropospheric response (Kuroda 2008; Yoshida and Yamazaki 2011); however, as can be seen in Fig. 4, the changes in tropospheric upwelling are not directly connected with the stratospheric circulation. The changes in tropospheric upwelling are driven by changes in convective activity, which are triggered by anomalously low temperatures near $100 \mathrm{hPa}$. Variations in tropopause temperatures associated with tropical waves are known to be capable of modulating equatorial convective activity (Zhou and Holton 2002; Suzuki et al. 2010). A similar process could explain the impact of cooling in the equatorial stratosphere on the troposphere during SSW events.

In the present global model experiments, convection is represented by a cumulus parameterization (Arakawa and Schubert 1974). The model sensitivity could depend significantly on the convective parameterization used. It will therefore be necessary to perform similar experiments using different models at different resolutions and with different convective parameterizations for more quantitative discussion.

Another notable result of the present experiment is a demon- stration of long duration of the influence of the extratropical initial conditions on the tropical circulation, which is attributable to the involvement of stratospheric processes. This information may be valuable for improving the extended two weeks weather forecasts in the tropics.

\section{Acknowledgements}

The authors thank to N. Eguchi for useful discussions.

\section{References}

Arakawa, A., and W. H. Schubert, 1974: Interaction of cumulus cloud ensemble with the large scale environment. Part I. J. Atmos. Sci., 31, 674-701.

Dunkerton, T. J., C.-P. F. Hsu, and M. E. McIntyre, 1981: Some Eulerian and Lagrangian diagnostics for a model stratospheric warming. $J$. Atmos. Sci., 38, 819-843.

Eguchi, N., and K. Kodera, 2010: Impacts of stratospheric sudden warming on tropical clouds and moisture fields in the TTL: A case study. SOLA, 6, 137-140.

Fritz, S., and S. D. Soules, 1970: Large-scale temperature changes in the stratosphere observed from Nimbus III. J. Atmos. Sci., 27, 10911097.

Garcia, R. R., 1987: On the meridional circulation of the middle atmosphere. J. Atmos. Sci., 44, 3599-3609.

Hsu, H.-H., B. J. Hoskins, and F.-F. Jin, 1990: The 1985/86 intraseasonal oscillation and the role of the extratropics. J. Atmos. Sci., 47, 823839 .

Inaba, M., and K. Kodera, 2010: Forecast study of the cold December of 2005 in Japan: Role of Rossby waves and tropical convection. $J$. Meteor. Soc. Japan, 88, 719-735.

Julian, P. R., and K. B. Labitzke, 1965: A study of atmospheric energetics during the January-February 1963 stratospheric warming. J. Atmos. Sci., 22, 597-610.

Kodera, K., 2006: Influence of stratospheric sudden warming on the equatorial troposphere. Geophys. Res. Lett., 33, L06804, doi:10.1029/2005 GL024510.

Kodera, K., N. Eguchi, J.-N. Lee, Y. Kuroda, and S. Yukimoto, 2011: Sudden changes in the tropical stratospheric and tropospheric circulation during January 2009. J. Meteor. Soc. Japan, 89, 283-290.

Kuroda, Y., 2008: Effect of stratospheric sudden warming and vortex intensification on the tropospheric climate. J. Geophys. Res., 113, D15110, doi:10.1029/2007JD009550.

Mizuta, R., K. Oouchi, H. Yoshimura, A. Noda, K. Katayama, S. Yukimoto, M. Hosaka, S. Kusunoki, H. Kawai, and M. Nakagawa, 2006: 20-km-mech global climate simulations using JMA-GSM model: Mean Climate States. J. Meteor. Soc. Japan, 84, 165-185.

Mukougawa, H., H. Sakai, and T. Hirooka, 2005: High sensitivity to the initial condition for the prediction of stratospheric sudden warming. Geophys. Res. Lett., 32, L17806, doi:10.1029/2005GL022909.

Mukougawa, H., T. Hirooka, T. Ichimaru, and Y. Kuroda, 2007: Hindcast AGCM experiments on the predictability of stratospheric sudden warming, Nonlinear Dynamics in Geosciences, A. A. Tsonis and J. B. Elsner, Eds., Springer-Verlag, New York, 221-233, 604 pp.

Norton, W. A., 2006: Tropical wave driving of the annual cycle in tropical tropopause temperatures. Part II: Model results. J. Atmos. Sci., 63, $1420-1431$.

Randel, W. J., 1993: Global variation of zonal mean ozone during stratospheric warming events. J. Atmos. Sci., 50, 3308-3321.

Suzuki, J., M. Fujiwara, A. Hamada, Y. Inai, J. Yamaguchi, R. Shirooka, F. Hasebe, and T. Takano, 2010: Cloud-top height variability associated with equatorial Kelvin waves in the tropical tropopause layer during the Mirai Indian Ocean cruise for the Study of the MJOconvection Onset (MISMO) campaign. SOLA, 6, 97-100.

Taguchi, M., and D. L. Hartmann, 2006: Increased occurrence of stratospheric sudden warmings during El Niño as simulated by WACCM. J. Climate, 19, 324-332.

Yoshida, K., and K. Yamazaki, 2011: Tropical cooling in the case of stratospheric sudden warming in January 2009: focus on the tropical tropopause layer. Atmos. Chem. Phys., 11, 6325-6336.

Zhou, X., and J. R. Holton, 2002: Intraseasonal variations of tropical coldpoint tropopause temperatures. J. Climate, 15, 1460-1473.

Manuscript received 5 October 2011, accepted 24 November 2011

SOLA: http://www.jstage.jst.go.jp/browse/sola 